\title{
Genomic signature of highland adaptation in fish: a case study in Tibetan Schizothoracinae species
}

\author{
Chao Tong ${ }^{1,2,3^{*}}$ D. Fei $\operatorname{Tian}^{1}$ and Kai Zhao ${ }^{1 *}$
}

\begin{abstract}
Background: Genome-wide studies on highland adaptation mechanism in terrestrial animal have been widely reported with few available for aquatic animals. Tibetan Schizothoracinae species are ideal model systems to study speciation and adaptation of fish. The Schizothoracine fish, Gymnocypris przewalskii ganzihonensis had underwent the ecological niche shift from salt water to freshwater, and also experienced a recent split from Gymnocypris przewalskii przewalskii. In addition, G. p. ganzihonensis inhabited harsh aquatic environment including low temperature and hypoxia as well as other Schizothoracinae species, its genetic mechanism of highland adaptation have yet to be determined.

Results: Our study used comparative genomic analysis based on the transcriptomic data of G. p. ganzihonensis and other four fish genome datasets to investigate the genetic basis of highland adaptation in Schizothoracine fish. We found that Schizothoracine fish lineage on the terminal branch had an elevated dN/ dS ratio than its ancestral branch. A total of 202 gene ontology (GO) categories involved into transport, energy metabolism and immune response had accelerated evolutionary rates than zebrafish. Interestingly, we also identified 162 genes showing signature of positive selection (PSG) involved into energy metabolism, transport and immune response in G. p. ganzihonesis. While, we failed to find any PSG related to hypoxia response as previous studies.

Conclusions: Comparative genomic analysis based on G. p. ganzihonensis transcriptome data revealed significant genomic signature of accelerated evolution ongoing within Tibetan Schizothoracinae species lineage. Molecular evolution analysis suggested that genes involved in energy metabolism, transport and immune response functions in Schizothoracine fish underwent positive selection, especially in innate immunity including toll-like receptor signaling pathway genes. Taken together, our result as a case study in Schizothoracinae species provides novel insights in understanding the aquatic animal adaptation to extreme environment on the Tibetan Plateau, and also provides valuable genomic resource for further functional verification studies.
\end{abstract}

Keywords: Comparative genomics, Schizothoracinae, Highland adaptation, Positive selection, Innate immunity

\section{Background}

It is of evolutionary interest to understand that how wildlife adapts to high altitude [1]. With an average elevation above $4000 \mathrm{~m}$, the Tibetan Plateau (TP) is one of the earth's most significant continental-scale highlands [2] imposes an extremely inhospitable environment on most

\footnotetext{
*Correspondence: tongchao1990@gmail.com; zhaokai@nwipb.cas.cn 'Key Laboratory of Adaptation and Evolution of Plateau Biota, Qinghai Key Laboratory of Animal Ecological Genomics, Laboratory of Plateau Fish Evolutionary and Functional Genomics, Northwest Institute of Plateau Biology, Chinese Academy of Sciences, Xining 810001, China

Full list of author information is available at the end of the article
}

wildlife, including hypoxia, high ultraviolet radiation and low temperatures $[3,4]$. Past research had indicated the adaptation of local wildlife to harsh living challenges. Recent studies employing genome-wide approaches on Tibetan terrestrial animal have primarily focused on response to hypoxia and energy metabolic pathways, including yak [4], Tibetan antelope [5], ground tit [2], Tibetan mastiff [6], Tibetan dog [7], Tibetan chicken [8]. Nevertheless, we know little about the mechanism of Tibetan aquatic animal adaptation to aquatic environment on the TP. Specifically, genetic mechanisms of adaptation in Schizothoracine fish have yet to be determined. 
Therefore, it may provide novel insights for understanding the mechanism of highland adaptation of Tibetan wildlife.

The Schizothoracinae is the largest and most diverse taxon of the TP ichthyofauna, which are distributed throughout the TP and its peripheral regions [9, 10]. Past research had revealed that Schizothoracinae species had well adapted to the harsh aquatic environment on the TP, including hypoxia, low temperature and even high salinity $[9,11-13]$, making them excellent models for investigating the genetic mechanism of aquatic animal adaptation to the extreme environment at high altitude. In addition, increasing studies focused on the speciation mechanism of the Schizothoracine fish and the uplift of the Tibetan Plateau [1417]. A Schizothoracine fish, Gymnocypris przewalskii ganzihonensis is the only fish inhabiting the Ganzi River (Fig. 1a). Another Schizothoracine fish, Gymnocypris przewalskii przewalskii is also the only fish inhabiting the Lake Qinghai (the largest salt lake in China). Previous research had indicated that the Ganzi River once flowed into the Lake Qinghai before the Wei-Jin-Nanbei Dynasty (200 to 589 A.D) [18]. An additional survey had revealed that the Ganzi River had disconnected to the Lake Qinghai as offshore great sand dune movement and shrinking of lake shoreline, which resulted in G. P. przewalskii colonized the freshwater habitat [18]. In addition, the taxonomists named this fish species as G. p. ganzihonensis based on morphological data [9], and also have been supported by mitochondrial evidences [17, 19-21]. Obviously, G. p. ganzihonensis had undergone the transition from salt water to freshwater, and this species also faced the challenges due to the low temperature and hypoxia environment in accord with other Schizothoracinae species. Therefore, it is an interesting issue to investigate the highland adaptation in fish species using G. p. ganzihonesis as a case study in Schizothoracinae.

Recent advances in sequencing technologies have offered the opportunity to map and quantify transcriptome in almost any species of interest that do not currently have a reference genome [22]. Noteworthy, most Schizothoracinae species are polyploidy, tetraploid, and even sixteen-ploid [9]. Transcriptome sequencing technology have been successfully applied in many polyploidy cases [23, 24], which is a rapid and effective approach to obtain massive protein-coding genes and molecular markers. This technology could facilitate investigations into the genetic basis of adaptations. Here we sequenced and generated the transcriptome of G. $p$. ganzihonensis as a case study in Tibetan Schizothoracinae species. We then performed comparative genomic analysis together with other previously available fish

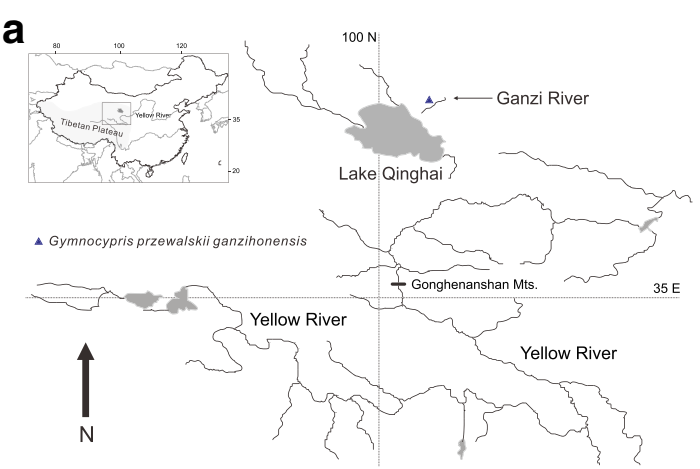

C

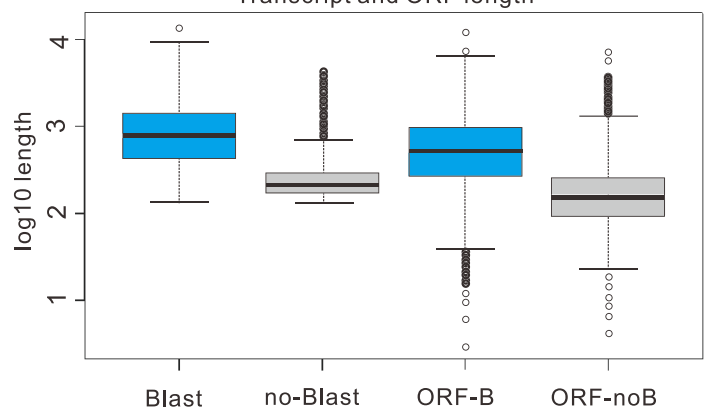

b

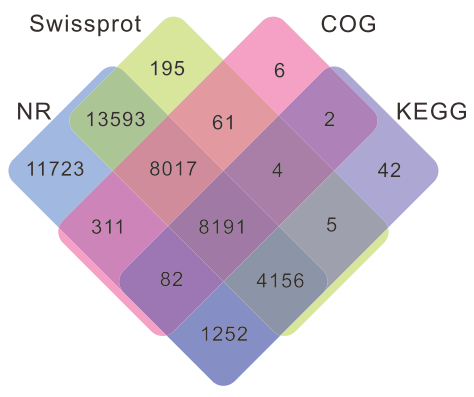

d

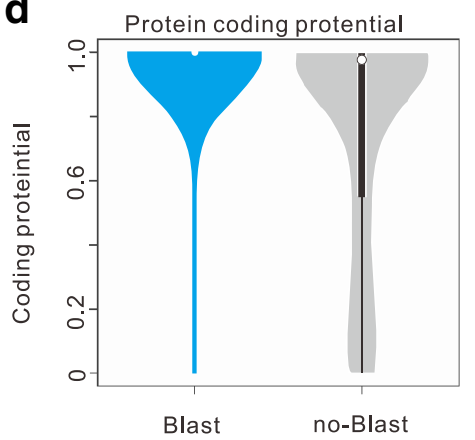

Fig. 1 Sampling site and annotation of G. p. ganzihonensis transcriptome. a The sampling map was created using the ArCGIS v10.1 (ESRI, CA, USA) and Adobe Illustrator CS5 (Adobe Systems Inc., San Francisco, CA). b Venn diagram shows shared and distinct genes under the annotations of NR, Swiss-Prot, COG and KEGG databases. Numbers indicating how many unigenes were annotated by each database. c Boxplot shows sequence characterization of the transcripts with and without detected homologs. $\mathbf{d}$ Protein coding potential were determined by CPAT and illustrated by boxplot 
genomes to reveal the potential genetic mechanism of highland adaptation in fish.

\section{Methods \\ Sample collection}

Ten adult G. p. ganzihonensis samples were captured and identified from the Ganzi River using gill nets. Gender was determined for each specimen. Next, four individuals (2 male and 2 female) were selected and dissected after anesthesia with MS-222 (Solarbio, Beijing, China). Gill and kidney from each individual were collected respectively, and then immediately stored in liquid nitrogen at $-80^{\circ} \mathrm{C}$.

\section{RNA extraction and transcriptome sequencing}

Total RNAs ( $n=8,4$ gills and 4 kidney) of two tissues were isolated from each of four individuals using TRIzol (Invitrogen, Carlsbad, CA) according to the manufacturer's instructions. The quantity and quality of total RNA was verified by an Agilent 2100 bioanalyzer (Agilent Technologies, Palo Alto, CA) and gel electrophoresis. Approximately $10 \mu \mathrm{g}$ of each RNA of same tissue from different individual were pooled for transcriptome library preparation (totally two independent libraries, gill and kidney), and sequenced on an Illumina $\mathrm{HiSeq}^{\mathrm{Tm}} 2000$ platform (parameters: 101-bp paired-end reads, 1 lane).

\section{Transcriptome assembly and annotation}

RNA-seq raw reads from each library were preprocessed to filter residual adapter sequences and low-quality reads $(\mathrm{Q}<20)$, Then all clean reads were assembled using the Trinity v2.2.0 program (https://github.com/trinityrnaseq/trinityrnaseq/releases) with default parameters. Contigs from each sample's assembly were clustered by CD-HIT program [25] (percent identity: 80\%; word size: 5) to generate a set of non-redundant unigenes, with a minimum overlap length of $200 \mathrm{bp}$. The assembled unigene sequences were aligned with a Blast- $X$ search (cut-off E-value of $1 \times 10^{-10}$ ) in public NCBI nonredundant (NR), Swiss-Prot, Cluster of Orthologous Groups (COG) databases and Kyoto Encyclopedia of Genes and Genomes (KEGG) database. Gene ontology (GO) terms were obtained from NR hits using Blast2GO (version_3.2) [26] with default parameters. Next, the Getorf program in EMBOSS (version_6.4.0) [27] was applied to obtain the Open reading frames (ORFs) of G. p. ganzihonensis genes. The CPAT tool [28] was used to predict the protein-coding potential for the assembled unigenes, with previously downloaded zebrafish dataset (Zv9/danRer7) as the assembly database and 0.38 as the coding probability cutoff.

\section{Orthologs identification}

Orthologs between Schizothoracine fish (G. p. ganzihonensis) and zebrafish were identified using reciprocal
BLAST best-hit method with an $\mathrm{E}$ value cutoff of $1 \times 10$ -10 as used in prior investigations [29]. Then 1:1 orthologs between four fish genomes, including zebrafish (Danio rerio), fugu (Takifugu rubripes), medaka (Oryzias latipes), and spotted gar (Lepisosteus oculatus) were obtained from Ensembl server using BioMART (JCI_4.2.75) [30]. Noteworthy, only the longest transcript was considered if one gene had multiple transcripts. Each orthologous gene set was aligned using PRANK [31] (parameters: $-\mathrm{f}=$ fasta $-\mathrm{F}$ -codon -noxml -notree -nopost) and trimmed using GBlocks [31] (parameters: $-\mathrm{t}=\mathrm{c}-\mathrm{b} 3=1-\mathrm{b} 4=6-\mathrm{b} 5=\mathrm{n}$ ). Then we deleted all gaps and " $N$ " from the alignments to lower the effect of ambiguous bases on the inference of positive selection. After deletion process, trimmed alignments shorter than 150 bp after removing sites with ambiguous data were discarded for subsequent analyses.

\section{Molecular evolution analyses}

The CODEML program in PAML 4.7a [32] with the freeratio model (parameters: model $=1$, NSsites $=0$, fix_omega $=0$, omega $=1$ ) was run on each ortholog, a concatenation of all alignments of the orthologs, and 1000 concatented alignments constructed from 150 randomly chosen orthologys, according to previous studies [29, 33-35]. The parameters of nonsynonymous (Ka or $\mathrm{dN}$ ), synonymous (Ks or $\mathrm{dS})$ and especially the substitution rate $(\omega=\mathrm{Ka} / \mathrm{Ks}$ or $\mathrm{dN} /$ dS) were used to meansure the lineage-specific evolutionary rates of above fish species. Based on GO term date which downloaded from BioMART (Ensembl, JCI_4.2.75), the orthologs were clustered into different functional GO terms and $d N, d S$ and $d N / d S$ ratio for each term was calculated, respectively. Finally, only GO categories with more than 20 orthologs were considered in this section analysis.

In addition, the CODEML program in PAML 4.7a with the branch-site model [36] (parameters: Null hypothesis: model $=2$, NSsites $=2$, fix_omega $=1$, omega $=1$ ) was used to identify positively selected genes (PSGs) in the Schizothoracine fish lineages, with other lineages being specified as the foreground branch. A LRT was constructed to compare a model that allows sites to be under positive selection $(\omega>1)$ on the foreground branch with the null model in which sites may evolve neutrally $(\omega=1)$ and under purifying selection $(\omega<1)$ with a posterior probability in excess of 0.95 based on the Bayes empirical Bayes (BEB) results [37]. Finally, the $P$ values were computed based on rigorous Chi-square statistic adjusted by FDR method and genes with adjusted $P$ value $<0.05$ were treated as candidates under positive selection.

\section{Results}

Transcriptome sequence analysis and assembly

Two pooled cDNA libraries derived from gill and kidney tissues of Schizothoracine fish, G. p. ganzihonensis were prepared and sequenced, totally generated 85,371,306 (gill) 
and 88,787,918 (kidney) raw 101-bp paired-end (PE) reads, respectively. After trimming adapters and removing lowquality reads, a total of 78,605,558 (gill) and 80,382,460 (kidney) clean reads were obtained from gill and kidney dataset, respectively. Finally, a total of 132,554 unigenes ranged from 201 to $16,310 \mathrm{bp}$, with an average length of 952 bp and an N50 of 1836 bp (Additional file 1: Table S1), the length distribution of all transcripts is shown in Additional file 2: Figure S1.

\section{Functional annotation}

To comprehensively annotate the transcriptome of G. $p$. ganzihonensis, all unigenes were queried against several public databases. A total of 94,321 (71.15\%) sequences yielded at least one significant match to an existing gene model in Blast-X search (Fig. 1b, Additional file 3: Table S2). Statistics results of COG and GO classification of all annotated unigenes were shown in Additional file 4: Figure S2 and Additional file 5: Figure S3. Almost half $(47.82 \%, n=63,404)$ of homologs aligned to known proteins have identified between $80 \%$ and $100 \%$. Due to fact of G. p. ganzihonensis is phylogenetically closer to zebrafish than some other fish species with complete genomic resources, it is not surprising that $81.72 \%(n=51,812)$ of the best hits were similar with model organism zebrafish (Additional file 6: Table S3). Next, the assembly unigene dataset was divided into two subsets to characterize the sequence features in detail, including unigenes with and without protein homology in NR, namely "Blast" and "no-Blast" respectively. The "Blast" subset had significantly larger unigenes length and longer ORFs than the "no-Blast" subset with $P$ value $<2.2 \times 10^{-5}$ in Wilcoxon rank sum test (Fig. $1 \mathrm{c}$ ). In addition, further analysis of the potential for protein coding with CPAT tool showed a significantly lower proteincoding potential in the "no-Blast" subset with $P$ value $<2.2 \times 10^{-6}$ (Fig. 1d).

\section{Accelerated evolution of the Schizothoracine fish lineage}

We identified the single-copy orthologs in Schizothoracine fish dataset and zebrafish, fugu, medaka, and spotted gar genome databases, resulting in a total of 6829 orthologs. Next, we used the species tree [38] in conjunction with a branch model constructed in PAML to determine $\mathrm{dN}, \mathrm{dS}$, and $\omega$ values across all 6829 orthologous genes. The result showed that the averaged $\omega$ value was significantly higher than other fish branches with $P$ $<2.2 \times 10-16$ in Wilcoxon rank sum test (Fig. 2a), implied that accelerated function evolution in Schizothoracine fish lineages. In addition, we analyzed the $\omega$ value for each branch for a concatenated alignment of all 6829 orthologs and 1000 concatenated alignments constructed from 150 randomly chosen orthologs. Intriguingly, using both comparison strategies, we found that Schizothoracine fish lineage exhibited a significantly higher $\omega$ value than other four fish branches in our study $(P<2.2 \times 10-16)$ (Fig. $2 \mathrm{~b}$ and $\mathrm{c})$. Here showed a clear clue was that the Schizothoracine fish branch had an elevated $\omega$ value than its ancestral branch and trend to ongoing accelerated evolution under the extreme environment on the TP (Fig. 2a).

After a strict filtering analysis, we calculated the mean $\omega$ value for each GO category with at least 20 orthologs in Schizothoracine fish and zebrafish lineages, respectively. A total of $202 \mathrm{GO}$ categories showing accelerated evolutionary rate $(P<0.05$, binomial test) were detected in Schizothoracine fish and 113 in zebrafish (Fig. 3 and Additional file 7: Table S4), which also confirmed overall accelerated evolution in Schizothoracine fish lineage. We then focused on these accelerated categories potentially associated with highland adaptation. Interestingly, these GO categories were mainly involved into four functional groups. One group was mostly related to biotic and abiotic stress, such as "response to DNA damage stimulus" and "activation of immune response". As the fact is that G. p. ganzihonensis in fresh water is split from its ancestor G. P. przewalskii in salt water, one groups was related to transport function, such as "ion transport" and "lipid transport". The other two groups involved in energy metabolism and immune system, such as "regulation of lipid metabolic process" and "regulation of immune system process" (Fig. 3 and Additional file 7: Table S4).

\section{Candidate genes under positive selection in Schizothoracine fish}

To better understand the potential genes contributed to Schizothoracine fish adaptation to TP, we used branchsite model in PAML to identify candidate positively selected genes (PSGs) in Schizothoracine fish lineage. After applying strict filtering criteria, we totally identified 162 PSGs $(P<0.05)$ in G. p. ganzihonensis (Additional file 8: Table S5). Intriguingly, the PSGs were also had functions associated with three main groups. The first functional group were related to transport functions, including solute carrier family 12 , member 1 (SLC12A1), solute carrier family 7, member 2 (SLC7A2), solute carrier family 38 , member 4 (SLC38A4). The PSGs in second group were associated with energy metabolism, including $N A D H$ dehydrogenase 1 (ND1), ATPase family, AAA domain containing 2 (ATAD2), ADP-ribosylation factor 3 (ARL3). Innate immunity function group was the third one, such as toll-like receptor 3 (TLR3), interferon regulatory factor 8 (IRF8), interleukin 10 (IL10) and tumor necrosis factor receptor superfamily, member $1 b$ (TNFRSF1b (Fig. 4a). Noteworthy, two infectious diseases "white spot" disease and saprolegniasis have been considered as the chief culprits and suffered high mortality rate of Schizothoracine fish 


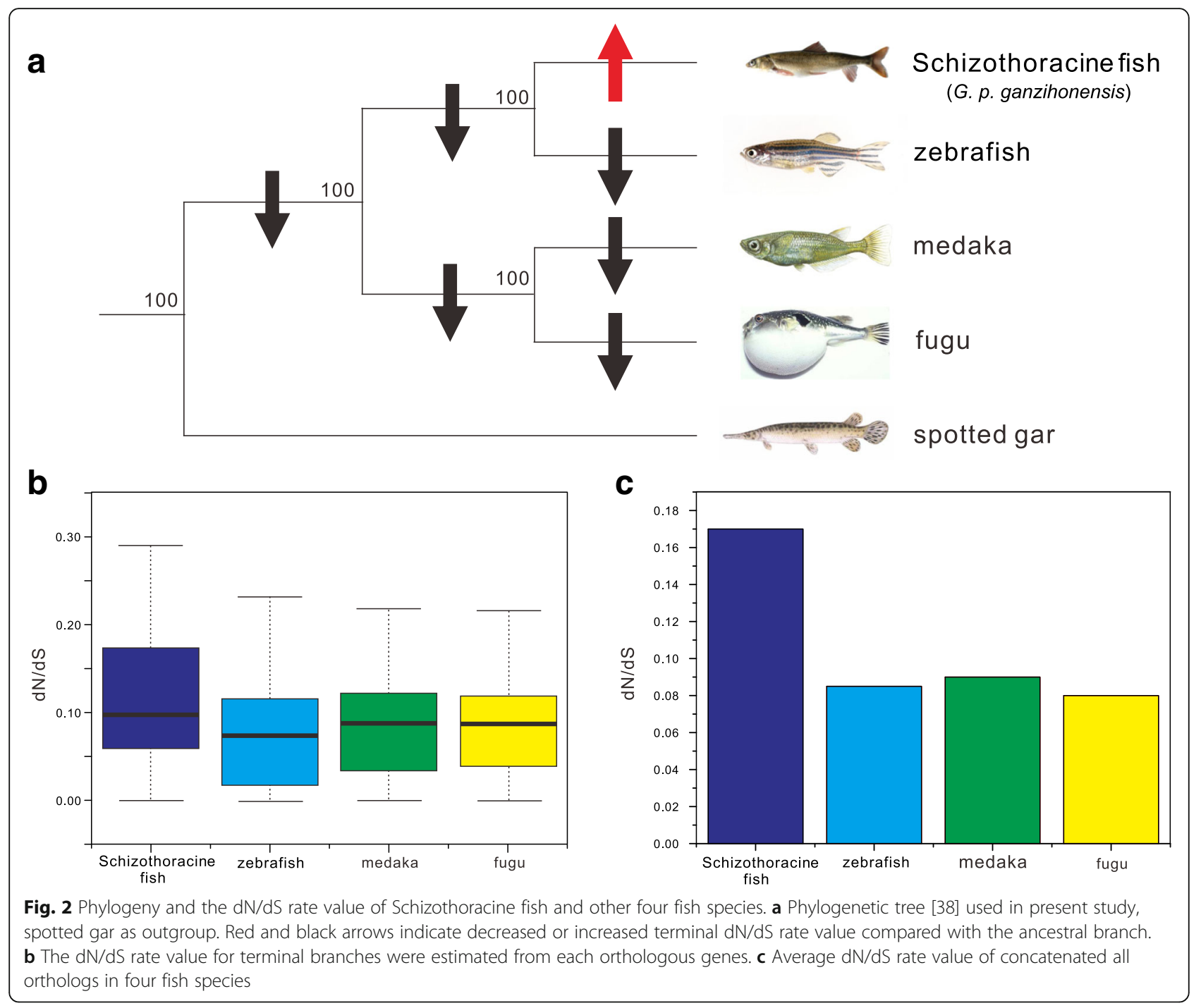

when in aquaculture industry rather than native environment (Fig. 4b). This led us to hypothesize whether there is a link between weak immune ability and PSGs in innate immunity. In addition, ten of the candidate PSGs were identified and linked to energy metabolism, including ATP13a, ABCC2a, ATAD2 and MRPL45 (Additional file 8: Table S5). This finding also confirmed that GO category of energy metabolism in G. p. ganzihonensis lineage may undergo accelerated evolution.

\section{Discussion}

In evolutionary biology, comparative genomic analysis had been widely applied in understanding the genetic basis of organisms' speciation [39-41] and adaptation [2, $4,8,11,29,35]$. Although whole genome sequencing data of nonmodel organisms have increasingly become available, most organisms still lack genomic resource. Transcriptome sequencing is an effective and accessible approach to initiate comparative genomic analysis on nonmodel organisms, because it could also contain a large number of protein-coding genes likely enriched for targets of natural selection. In this study, we sequenced and annotated the transcriptome of the Schizothoracine fish, G. p. ganzihonensis [9, 18], and identified more than 6000 pairwise orthologs among five fish genomes. Then, we performed comparative genomic analysis on this Schizothoracine fish using its de novo assembly transcriptome dataset and other four fish genomes. Finally, this transcriptome resource could develop our understanding of genetic makeup of highland fishes and provide a foundation for further studies to identify candidate genes underlying adaptation to the Tibetan Plateau of Schizothoracine fishes.

How an organism adapts to environment change is an important issue in evolutionary biology [42]. Adaptive evolution may prefer to proceed at molecular level, expressed by an increase in ratio of nonsynonymous substitutions to synonymous substitutions [43]. Previous 


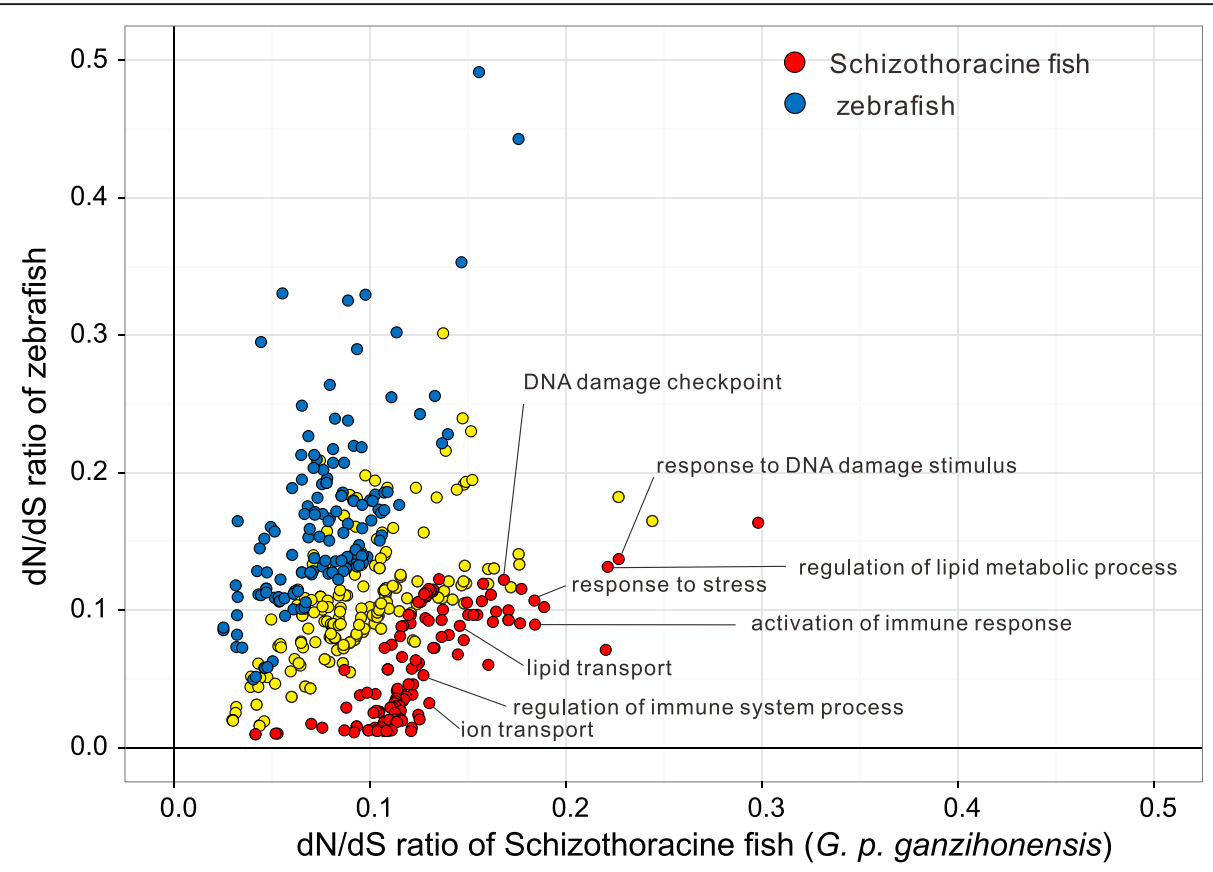

Fig. 3 Accelerated evolution within GO category. Scatter plot of mean dN/dS rate value for each GO category in Schizothoracine fish and zebrafish lineages. GO categories with significantly higher mean dN/dS rate value in Schizothoracine fish (red) and zebrafish (blue) are highlighted, respectively. Yellow points represent $\mathrm{GO}$ categories with higher but not statistically significant mean dN/dS rate value in both lineages

studies revealed that terrestrial organisms adapted to life at high altitude by gene family expansion, accelerated evolutionary rate and underwent positive selection on genes associated with specific function $[2,4,7,8]$. Convergence is an independent evolution of similar physiological or morphological features in different species [44].
Its occurrence could support the hypothesis that specific ecological environment challenges can induce species to evolve in predictable and repeatable ways [45]. Our current analysis results suggested that Schizothoracine fish lineage trends to genome-wide accelerated evolution relative to other fish lineages. Past evidence indicated that accelerated

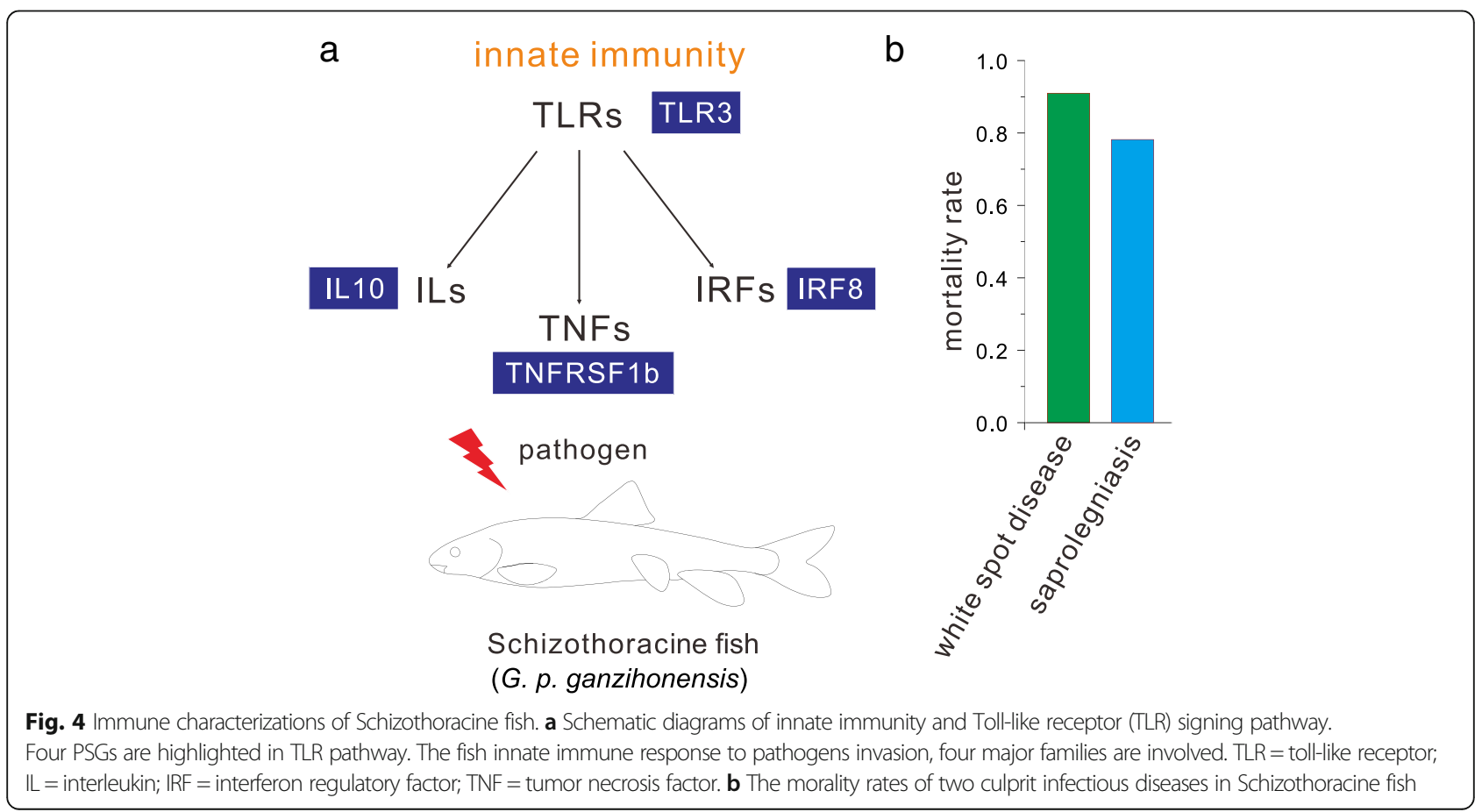


evolution is usually driven by positive selection [32], we therefore speculated that Schizothoracine fish may adaptively speed up its evolutionary rate of genes for better adaptation to extreme environment of the TP. The relaxation of function constraint could possibly trigger accelerated evolution, which the hypothesis should need more cases based on population genomic analyses to support. Furthermore, compared with the ancestral branch, the terminal branch of Schizothoracine fish had underwent an elevated $\mathrm{dN} / \mathrm{dS}$ ratio, implying that accelerated evolution only in the Schizothoracine fish lineage after diverged from zebrafish (also belonged to Cyprinidae). Previous studies had identified various adaptive processes that may be responsible for highland adaptation in terrestrial animal, including energy metabolism and hypoxia response $[2,4,46]$. Therefore it is not surprising that many GO categories related to energy metabolism and stress response in aquatic animal, Schizothoracine fish. A striking finding of the present study is that "transport function" genes may undergo accelerated evolution, this is consisted of the finding in recent study on the extremely alkaline environment adaptation mechanism in Amur ide, Leuciscus waleckii [47]. This finding implied that the adaptive evolution might play important role in this recent split Schizothoracine fish, G. p. ganzihonesis in transition of salt water to fresh water.

The functions of candidate PSGs were consistent with above identified functional groups of GO categories exhibiting accelerated evolution. Recent studies revealed the genetic basis of terrestrial animal adaptation to low oxygen and low temperature environment at high altitude $[1,2,4,7,12$, 13, 29]. In G. p. ganzihonesis, we failed to identify any PSG involved in hypoxia response. This may because the oxygen condition in Tibetan Plateau aquatic environment is different with ground. Previous evidence indicated that abundant and diverse of hydrophyte species in Ganzi River [18], these factors could have positive impacts on the dissolved oxygen content as the plant photosynthesis, which may help to explain the absence of PSGs related to response to hypoxia function. Low temperature is a typical feature of lake and river environment on the TP, which faced up this challenge for all aquatic animals. Accord to previous findings [2, 4], several candidate PSGs involved in energy metabolism were identified. For example, the ATP13a gene that encodes an accessory protein for ATP synthesis and decomposition, suggesting an important role in energy metabolism to adaptation to this low temperature water environment. In addition, SLC family play vital roles in transport function contribute to organism response to dynamic aquatic environment [48]. We also identified several SLC family members shown positive selection in Schizothoracine fish lineage, such as SLC12A1, SLC7A2, SLC38A4. This finding were similar to previous genome-wide study on the Amur ide in extremely alkaline environment [47], which indicated that adaptive evolution within genes involved in transport function contribute to provide novel insight into adaptation to extreme aquatic environment on the Tibetan Plateau. Remarkably, here we provide another novel insight to understand the genetic mechanism of highland adaptation in Schizothoracine fish, the adaptive evolution of innate immunity. Recent evidence showed that Schizothoracine fish is susceptible to infectious diseases and triggered high mortality rate in non-native environment [49-52]. In addition, recent genome-wide study reveal that adaptive evolution of innate immunity contributed to fish well response to pathogen invasion [53]. Intriguingly, we identified significant positive selection signs in TLR3, IRF8, IL10 and TNFRSF1b involved in innate immunity. This finding is similar with our previous reports on the PSGs and neofunctionalization in TLR signaling pathway genes $[49,51,52]$, suggesting that adaptive evolution of innate immunity may play important roles in Schizothoracine fish adaptation to high attitude aquatic life.

\section{Conclusions}

In summary, we have sequenced and annotated the first transcriptome of a recent split Schizothoracine fish, G. p. ganzihonensis. Comprehensive analyses of over 6000 orthologs among G. p. ganzihonensis and four fish genome databases identified evidence for sign of accelerated evolution in G. p. ganzihonensis lineage and only the terminal branch of G. p. ganzihonensis had an elevated dN/ dS ratio than ancestral branches. Number of GO categories involved into energy metabolism, transport and immune response showed rapid evolution in compared with model zebrafish. Intriguingly, we found that many genes showing signature of positive selection in G. $p$. ganzihonensis lineage were enriched in functions associated with energy metabolism, and a novel finding is that PSGs also related to innate immunity, especially in tolllike receptor signaling pathway. While we failed to identify any PSG involved in hypoxia response as previous studies reported in terrestrial animal and several Tibetan fish species. This transcriptome dataset also provides a valuable resource for further functional verification study which will develop our understanding of ecological and evolutionary questions concerning fish species on the TP.

\section{Notes}

Chao Tong and Fei Tian contributed equally to this work.

\section{Additional files}

Additional file 1: Table S1. Summary of sequencing, assembly and analysis of G. p. ganzihonensis transcriptome. (DOC $36 \mathrm{~kb}$ ) 
Additional file 2: Figure S1. Length distribution of all transcripts. Transcripts of gill and kidney datasets are calculated respectively. Cumulative length of unigenes is also calculated. (PDF $138 \mathrm{~kb}$ )

Additional file 3: Table S2. Annotation of assembled unigenes in G. $p$. ganzihonensis transcriptome. (XLS $41915 \mathrm{~kb}$ )

Additional file 4: Figure S2. COG classification of assembled unigenes in G. p. ganzihonensis transcriptome. (PDF $22 \mathrm{~kb}$ )

Additional file 5: Figure S3. $G O$ classification of assembled unigenes in G. p. ganzihonensis transcriptome. (PDF $21 \mathrm{~kb}$ )

Additional file 6: Table S3. Species distribution is calculated as a percentage of the total homologous sequences. (XLS $27 \mathrm{~kb}$ )

Additional file 7: Table S4. GO categories showing accelerated evolutionary rates within G. p. ganzihonensis and Danio rerio. (XLS 104 kb)

Additional file 8: Table S5. Positively selected genes (PSGs) identified in G. p. ganzihonensis. (XLS 64 kb)

\section{Abbreviations}

COG: Cluster of Orthologous Groups; FDR: false discovery ratio.; GO: Gene ontology; NR: non-redundant; ORFs: Open reading frames; PSG: Positively selected gene; TP: Tibetan Plateau

\section{Acknowledgements}

We thank Dr. Renyi Zhang, Yongtao Tang and Chenguang Feng in our group for critical comments on early manuscript. We also thank the editor and two anonymous reviewers for their valuable comments.

\section{Funding}

This work was supported by grants from the National Natural Science Foundation of China (30970341, 31572258 and 31700325), the Key Innovation Plan of Chinese Academy of Sciences (KSCX2-EW-N-004) and the Open Foundation from the Qinghai Key Laboratory of Tibetan Medicine Pharmacology and Safety Evaluation (2014-ZY-03).

\section{Availability of data and materials}

The raw reads produced in this study were deposited in the NCBI database Sequence Read Archive under the accession number SRX673793 and SRX673788.

\section{Authors' contributions}

$\mathrm{CT}$ and $\mathrm{KZ}$ conceived and designed the experiments. CT, FT and $\mathrm{KZ}$ collected the samples. CT analyzed the data and wrote the paper. All authors read and approved the final manuscript.

\section{Ethics approval and consent to participate}

All animal experiments were approved by the Animal Care and Use Committees of the Northwest Institute of Plateau Biology, Chinese Academy of Sciences.

\section{Consent for publication}

Not applicable.

\section{Competing interests}

The authors declare that they have no competing interests.

\section{Publisher's Note}

Springer Nature remains neutral with regard to jurisdictional claims in published maps and institutional affiliations.

\section{Author details}

'Key Laboratory of Adaptation and Evolution of Plateau Biota, Qinghai Key Laboratory of Animal Ecological Genomics, Laboratory of Plateau Fish Evolutionary and Functional Genomics, Northwest Institute of Plateau Biology, Chinese Academy of Sciences, Xining 810001, China. ${ }^{2}$ University of Chinese Academy of Sciences, Beijing 100049, China. ${ }^{3}$ Department of Biology, University of Pennsylvania, Philadelphia, PA 19104-6018, USA
Received: 30 November 2016 Accepted: 28 November 2017

Published online: 06 December 2017

\section{Reference}

1. Bickler PE, Buck LT. Hypoxia tolerance in reptiles, amphibians, and fishes: life with variable oxygen availability. Annu Rev Physiol. 2007;69:145-70.

2. Qu Y, Zhao H, Han N, Zhou G, Song G, Gao B, Tian S, Zhang J, Zhang R, Meng $X$. Ground tit genome reveals avian adaptation to living at high altitudes in the Tibetan plateau. Nat Commun. 2013:4

3. An Z, John EK, Warren LP, Stephen CP. Evolution of Asian monsoons and phased uplift of the Himalaya-Tibetan plateau since late Miocene times. Nature. 2001;411(6833):62-6.

4. Oiu O, Zhang G, Ma T, Oian W, Wang J, Ye Z, Cao C, Hu Q, Kim J, Larkin DM. The yak genome and adaptation to life at high altitude. Nat Genet. 2012:44(8):946-9.

5. Ge R, Cai Q, Shen Y, San A, Ma L, Zhang Y, Yi X, Chen Y, Yang L, Huang Y. Draft genome sequence of the Tibetan antelope. Nat Commun. 2013;4:1858.

6. Gou X, Wang Z, Li N, Qiu F, Xu Z, Yan D, Yang S, Jia J, Kong X, Wei Z. Wholegenome sequencing of six dog breeds from continuous altitudes reveals adaptation to high-altitude hypoxia. Genome Res. 2014;24(8):1308-15.

7. Wang G, Fan R, Zhai W, Liu F, Wang L, Zhong L, Wu H, Yang H, Wu S, Zhu $\mathrm{C}$. Genetic convergence in the adaptation of dogs and humans to the highaltitude environment of the Tibetan plateau. Genome Biology and Evolution. 2014:6(8):2122-8.

8. Wang M-S, Li Y, Peng M-S, Zhong L, Wang Z-J, Li Q-Y, Tu X-L, Dong Y, Zhu C-L, Wang L. Genomic analyses reveal potential independent adaptation to high altitude in Tibetan chickens. Mol Biol Evol. 2015;32(7):1880-889.

9. Wu Y, Wu C. The fishes of the Qinghai-Xizang plateau: Sichuan Publishing House of Science \& Technology; 1992.

10. Cao W, Chen Y, Wu Y, Zhu S. Origin and evolution of schizothoracine fishes in relation to the upheaval of the Qinghai-Tibetan plateau. Beijing: Science Press; 1981.

11. Zhang R, Ludwig A, Zhang C, Tong C, Li G, Tang Y, Peng Z, Zhao K. Local adaptation of Gymnocypris przewalskii (Cyprinidae) on the Tibetan plateau. Sci Rep. 2015;5:9780

12. Guan L, Chi W, Xiao W, Chen L, He S. Analysis of hypoxia-inducible factor alpha polyploidization reveals adaptation to Tibetan plateau in the evolution of schizothoracine fish. BMC Evol Biol. 2014;14(1):192.

13. Xu Q, Zhang C, Zhang D, Jiang H, Peng S, Liu Y, Zhao K, Wang C, Chen L. Analysis of the erythropoietin of a Tibetan plateau schizothoracine fish (Gymnocypris Dobula) reveals enhanced cytoprotection function in hypoxic environments. BMC Evol Biol. 2016;16(1):1.

14. Duan Z, Zhao K, Peng Z, Li J, Diogo R, Zhao X, He S. Comparative phylogeography of the Yellow River schizothoracine fishes (Cyprinidae): Vicariance, expansion, and recent coalescence in response to the quaternary environmental upheaval in the Tibetan plateau. Mol Phylogenet Evol. 2009:53(3):1025-31.

15. Zhao K, Duan ZY, Peng ZG, Guo SC, Li JB, He SP, Zhao XQ. The youngest split in sympatric schizothoracine fish (Cyprinidae) is shaped by ecological adaptations in a Tibetan plateau glacier lake. Mol Ecol. 2009;18(17):3616-28.

16. Zhao K, Duan Z, Peng Z, Gan X, Zhang R, He S, Zhao X. Phylogeography of the endemic Gymnocypris chilianensis (Cyprinidae): sequential westward colonization followed by allopatric evolution in response to cyclical Pleistocene glaciations on the Tibetan plateau. Mol Phylogenet Evol. 2011;59(2):303-10.

17. Zhang R, Peng Z, Li G, Zhang C, Tang Y, Gan X, He S, Zhao K. Ongoing speciation in the Tibetan plateau Gymnocypris species complex. PLoS One. 2013:8(8):e71331.

18. Zhu S, Wu Y. Study of fish fauna in Qinghai Lake. Beijing: Science Press; 1975

19. Qi D, Chao Y, Zhao L, Shen Z, Wang G. Complete mitochondrial genomes of two relatively closed species from Gymnocypris (Cypriniformes: Cyprinidae): genome characterization and phylogenetic considerations. Mitochondrial DNA. 2013;24(3):260-2.

20. Zhang R, Li G, Zhang C, Tang Y, Zhao K. Morphological differentiations of the gills of two Gymnocypris przewalskii subspecies in different habitats and their functional adaptations. Zool Res. 2013:34(4):387-91.

21. Tong C, Tang Y, Zhao K. The complete mitochondrial genome of Gymnocypris Przewalskii kelukehuensis (Teleostei: Cyprinidae). Conserv Genet Resour. 2017;9(3):443-5.

22. Wang Z, Gerstein M, Snyder M. RNA-Seq: a revolutionary tool for transcriptomics. Nat Rev Genet. 2009;10(1):57-63. 
23. Bancroft I, Morgan C, Fraser F, Higgins J, Wells R, Clissold L, Baker D, Long Y, Meng J, Wang $X$. Dissecting the genome of the polyploid crop oilseed rape by transcriptome sequencing. Nat Biotechnol. 2011;29(8):762-6.

24. Dong S, Adams KL. Differential contributions to the transcriptome of duplicated genes in response to abiotic stresses in natural and synthetic polyploids. New Phytol. 2011;190(4):1045-57.

25. Li W, Godzik A. Cd-hit: a fast program for clustering and comparing large sets of protein or nucleotide sequences. Bioinformatics. 2006;22(13):1658-9.

26. Conesa A, Götz S, García-Gómez JM, Terol J, Talón M, Robles M. Blast2GO: a universal tool for annotation, visualization and analysis in functional genomics research. Bioinformatics. 2005;21(18):3674-6.

27. Rice P, Longden I, Bleasby A. EMBOSS: the European molecular biology open software suite. Trends Genet. 2000;16(6):276-7.

28. Wang L, Park HJ, Dasari S, Wang S, Kocher J-P, Li W. CPAT: coding-potential assessment tool using an alignment-free logistic regression model. Nucleic Acids Res. 2013;41(6):e74

29. Yang L, Wang Y, Zhang Z, He S. Comprehensive transcriptome analysis reveals accelerated genic evolution in a Tibet fish, Gymnodiptychus pachycheilus. Genome Biology and Evolution. 2015;7(1):251-61

30. Durinck S, Moreau Y, Kasprzyk A, Davis S, De Moor B, Brazma A, Huber W. BioMart and bioconductor: a powerful link between biological databases and microarray data analysis. Bioinformatics. 2005:21(16):3439-40.

31. Löytynoja A, Goldman N. An algorithm for progressive multiple alignment of sequences with insertions. Proc Natl Acad Sci U S A. 2005;102(30):10557-62.

32. Yang Z. PAML 4: phylogenetic analysis by maximum likelihood. Mol Biol Evol. 2007;24(8):1586-91.

33. Wang $Y$, Yang L, Zhou K, Zhang Y, Song Z, He S. Evidence for adaptation to the Tibetan plateau inferred from Tibetan loach transcriptomes. Genome biology and evolution. 2015;7(11):2970-82.

34. Yang Y, Wang L, Han J, Tang X, Ma M, Wang K, Zhang X, Ren Q, Chen Q Qiu Q. Comparative transcriptomic analysis revealed adaptation mechanism of Phrynocephalus Erythrurus, the highest altitude lizard living in the Qinghai-Tibet plateau. BMC Evol Biol. 2015;15(1):101.

35. Tong C, Fei T, Zhang C, Zhao K. Comprehensive transcriptomic analysis of Tibetan Schizothoracinae fish Gymnocypris Przewalskii reveals how it adapts to a high altitude aquatic life. BMC Evol Biol. 2017;17(1):74.

36. Zhang J, Nielsen R, Yang Z. Evaluation of an improved branch-site likelihood method for detecting positive selection at the molecular level. Mol Biol Evol. 2005:22(12):2472-9.

37. Yang Z, Wong WS, Nielsen R. Bayes empirical Bayes inference of amino acid sites under positive selection. Mol Biol Evol. 2005:22(4):1107-18.

38. Near TJ, Eytan RI, Dornburg A, Kuhn KL, Moore JA, Davis MP, Wainwright PC, Friedman M, Smith WL. Resolution of ray-finned fish phylogeny and timing of diversification. Proc Natl Acad Sci. 2012;109(34):13698-703.

39. Fruciano C, Franchini P, Kovacova V, Elmer KR, Henning F, Meyer A. Genetic linkage of distinct adaptive traits in sympatrically speciating crater lake cichlid fish. Nat Commun. 2016;7

40. Elmer KR, Fan S, Kusche H, Spreitzer ML, Kautt AF, Franchini P, Meyer A. Parallel evolution of Nicaraguan crater lake cichlid fishes via non-parallel routes. Nat Commun. 2014:5

41. Brawand D, Wagner CE, Li Yl, Malinsky M, Keller I, Fan S, Simakov O, Ng AY, Lim ZW, Bezault E. The genomic substrate for adaptive radiation in African cichlid fish. Nature. 2014;513(7518):375-81

42. Smith NG, Eyre-Walker A. Adaptive protein evolution in drosophila. Nature. 2002;415(6875):1022-4

43. Bakewell MA, Shi P, Zhang J. More genes underwent positive selection in chimpanzee evolution than in human evolution. Proc Natl Acad Sci. 2007:104(18):7489-94.

44. Feil R, Berger F. Convergent evolution of genomic imprinting in plants and mammals. Trends Genet. 2007;23(4):192-9.

45. Stern DL. The genetic causes of convergent evolution. Nat Rev Genet. 2013;14(11):751-64.

46. Beall CM, Decker MJ, Brittenham GM, Kushner I, Gebremedhin A, Strohl KP. An Ethiopian pattern of human adaptation to high-altitude hypoxia. Proc Natl Acad Sci. 2002;99(26):17215-8.

47. Xu J, Li J-T, Jiang Y, Peng W, Yao Z, Chen B, Jiang L, Feng J, Ji P, Liu G, et al. Genomic basis of adaptive evolution: the survival of Amur ide (Leuciscus waleckii) in an extremely alkaline environment. Mol Biol Evol. 2016;34(1): $145-59$
48. Hediger MA, Romero MF, Peng JB, Rolfs A, Takanaga $H$, Bruford EA. The $A B C s$ of solute carriers: physiological, pathological and therapeutic implications of human membrane transport proteinsIntroduction. Pflugers Arch - Eur J Physiol. 2004:447(5):465-8.

49. Tong C, Lin Y, Zhang C, Shi J, Qi H, Zhao K. Transcriptome-wide identification, molecular evolution and expression analysis of toll-like receptor family in a Tibet fish, Gymnocypris przewalskii. Fish and Shellfish Immunology. 2015:46(2):334-45.

50. Tong C, Zhang C, Zhang R, Zhao K. Transcriptome profiling analysis of naked carp (Gymnocypris przewalskii) provides insights into the immunerelated genes in highland fish. Fish and Shellfish Immunology. 2015;46(2):366-77

51. Tong C, Tian F, Tang Y, Feng C, Guan L, Zhang C, Zhao K. Positive Darwinian selection within interferon regulatory factor genes of Gymnocypris przewalskii (Cyprinidae) on the Tibetan plateau. Fish and Shellfish Immunology. 2016;50:34-42.

52. Tong C, Zhao K. Signature of adaptive evolution and functional divergence of TLR signaling pathway genes in Tibetan naked carp Gymnocypris Przewalskii. Fish and Shellfish Immunology. 2016;53:124.

53. Wu C, Zhang D, Kan M, Lv Z, Zhu A, Su Y, Zhou D, Zhang J, Zhang Z, Xu M. The draft genome of the large yellow croaker reveals well-developed innate immunity. Nat Commun. 2014;5

\section{Submit your next manuscript to BioMed Central and we will help you at every step:}

- We accept pre-submission inquiries

- Our selector tool helps you to find the most relevant journal

- We provide round the clock customer support

- Convenient online submission

- Thorough peer review

- Inclusion in PubMed and all major indexing services

- Maximum visibility for your research

Submit your manuscript at www.biomedcentral.com/submit 